346

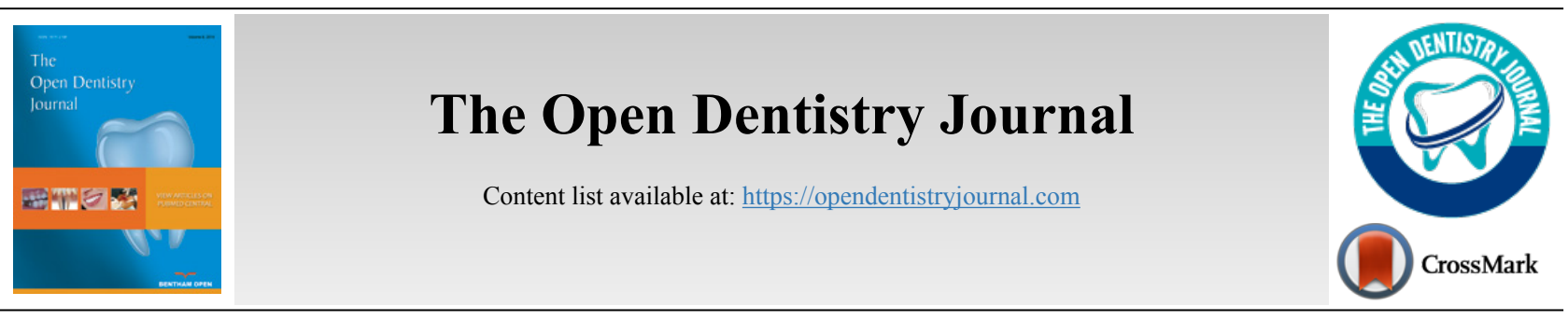

RESEARCH ARTICLE

\title{
Efficacy of Dental Implants Placements between Lateral and Crestal Sinus Lift Techniques: A Split-Mouth Study
}

\author{
Saad Al-Almaie ${ }^{1, *}$ and Abdul M. Kavarodi ${ }^{2}$ \\ ${ }^{1}$ Medical Administration, King Fahd Military Medical Complex, Dhahran, Saudi Arabia \\ ${ }^{2}$ Department of Dental Science, King Fahd Military Medical Complex, Dhahran, Saudi Arabia
}

\begin{abstract}
:
Background:

Bone atrophy and pneumatization of the maxillary sinus resulting from tooth loss, associated with low bone density in the region, do not usually allow adequate quantity and quality for the installation of osseointegrated implants.

\section{Objective:}

To evaluate the efficacy of crestal and lateral sinus lift techniques which are performed on same patients.

\section{Materials and Methods:}

Ten patients who required both crestal and lateral approaches in 20 sinus lifts were selected; 31 endosseous dental implants were placed into these patients, with a reported timeline for each approach. Assessment of the surgical procedures in all patients was done using a Visual Analog Scale (VAS). Clinical and radiographic parameters, including Pocket Probing Depth (PPD), distance between the implant shoulder and the mucosal margin (DIM) and distance between the implant shoulder and first visible bone-implant contact (DIB), were analyzed to compare implant survival for both approaches.

Results:

All implants were successfully osseointegrated. No significant differences were observed between lateral and crestal approaches in PPD, DIM and DIB ( $p$-values $=0.0504,0.7784$ and 0.18170 , respectively), and in the total VAS scores ( $p$-value $\geq 0.05$ ). Most of the patients preferred the crestal approach due to the delay in implant placements with the lateral approach.

\section{Conclusion:}

Evaluation of the sinus floor elevation is more precise when both techniques are performed on the same patient. Furthermore, although postoperative vertigo is a major concern with the crestal approach; patients preferred this approach over the lateral technique because it is less invasive and required less time for the procedure.
\end{abstract}

Keywords: Split mouth study, Sinus floor elevation, Vertigo, Lateral and crestal approaches, Visual analog scale, Reformatted fly-through image, Osteotome.

\section{INTRODUCTION}

An implant-supported dental prosthesis can be a viable treatment option when there is sufficient quantity and quality of bone. The limitations of dental implant placement in the posterior maxilla, due to alveolar ridge resorption and excessive maxillary sinus pneumatization, can be overcome by

Address correspondence to this author at Medical Administration, King Fahd Military Medical Complex, P.O.Box 946 zipcode Dhahran 31932, Saudi Arabia; Tel: +011966504805413; E-mail: stsralmaie@yahoo.com maxillary sinus floor elevation. Maxillary sinus floor elevation was initially described by Tatum at the Alabama Implant Conference in 1976 and was subsequently published by Boyne in 1980 [1 - 4]. Since its first description, numerous articles have been published in this field regarding different grafting materials, modifications to the classic technique and comparisons between different techniques [5 - 8].

Currently, two main approaches to the maxillary sinus floor elevation procedure are widely practiced. The first 
approach is the classic lateral antrostomy, and the second is the conservative Osteotome Sinus Floor Elevation (OSFE) [9]. Maxillary sinus elevation to facilitate the placement of dental implants (OSFE) has gained widespread acceptance because the technique is less invasive and less time consuming [9]. A residual alveolar ridge of $5 \mathrm{~mm}$ is the minimum bone height required to consider the OSFE technique over the lateral approach $[10,11]$. Discomfort during the infracture of the sinus floor, intra-operative or post-operative dizziness, tinnitus, disorientation, nausea, vomiting, and vasovagal syncope are the main complications specific to OSFE [12].

Patients requiring both OSFE and lateral antrostomy procedures on either side of the maxilla, as per current practice guidelines, constitute a very rare group. The quality and quantity of the maxillary residual ridge are the key factor in deciding between the two techniques in all patients [13]. Clinical and radiological assessments are utilized to evaluate the quality and quantity of the residual ridge. Both techniques have considerably variable procedural success rates, and patients' appraisal of the techniques can also vary considerably. Although many studies have compared and contrasted lateral window versus OSFE approaches for sinus lift procedures, a split-mouth study in the same patients has not yet been attempted. A split-mouth study, which is a self-controlled study design, is preferred because it eliminates sources of bias that occur in similar controlled studies [14 - 16]. Therefore, the aim of this study was to evaluate the efficacy of crestal and lateral sinus lift techniques which are performed on same patients.

\section{MATERIALS AND METHODS}

\subsection{Ethical Approval of the Study Protocol}

Approval for the study was granted by the Ethics Committee of the Medical Association for the Military Hospital. The committee met after the completion of the study and approved the study for publication.

All of the patients were informed about the aims and protocol of the study, and provided proper consent. All bilateral sinus lift cases in the center during the period from 2006 to 2012 were considered. Patients were selected according to the inclusion criteria, from a group of sixty patients recruited from the same center [17].

\subsection{Inclusion Criteria}

- Patients who required a bilateral sinus lift for implant treatment in the posterior maxilla.

- Patients who underwent an OSFE procedure without placing grafting material when the RBH was equal to or greater than $5 \mathrm{~mm}$.

- Patients who underwent a lateral sinus lift procedure if the RBS was a minimum of $2 \mathrm{~mm}$ and a maximum of 4 $\mathrm{mm}$.

- Patients who required a bilateral sinus lift, with one side indicated for OSFE and the other side for a lateral approach, as per the previous criteria.

- Patients who require at least $1 \mathrm{~mm}$ of bone on each side for implant stability.

- Patients free from any systemic or local contraindications for dental implant placement.

\subsection{Exclusion Criteria}

[1] Patients who required a bilateral sinus, in whom both the right and left sides were indicated for OSFE per current practice guidelines [9].

[2] Patients who required a bilateral sinus, in whom both the right and left sides were indicated for sinus lift by the lateral approach per current practice guidelines $[10$, $11]$.

Ten patients were selected out of the sixty patients previously recruited. Thirty-one dental implants were inserted into seven men and three women (Table 1). Their ages ranged between 38 and 68, with a mean age of 53 years old. These patients presented with bilateral edentulous spaces in the posterior maxilla with reduced residual bone height, making standard implant placement impossible. These patients have lost their teeth due to periodontal diseases, caries, trauma or endodontic failures. All of the patients were treated bilaterally via elevation of the sinus floor to place implants in the posterior maxilla, using both techniques chosen on a random basis by two operators. Pre-operative records included orthopantomography (Fig. 1), intraoral photographs and study casts. Computed tomography (CT) scans were obtained using SOMATOM Definition Flash (Siemens, Forchheim, Bavaria, Germany) with Syngo Dental CT software (Syngo Somaris/ 7 version VA 40A_10_P15, 2011). All the patients' images were obtained with SOMATOM Definition Flash software after the procedures. Local anesthesia was used for all patients. Soft tissue parameters were obtained to compare the peri-implant soft tissue conditions and marginal bone levels between the two approaches. The patients' appraisals of both techniques were assessed using a Visual Analog Scale (VAS) questionnaire. The survival of the placed implants was evaluated by standard measurements [18].

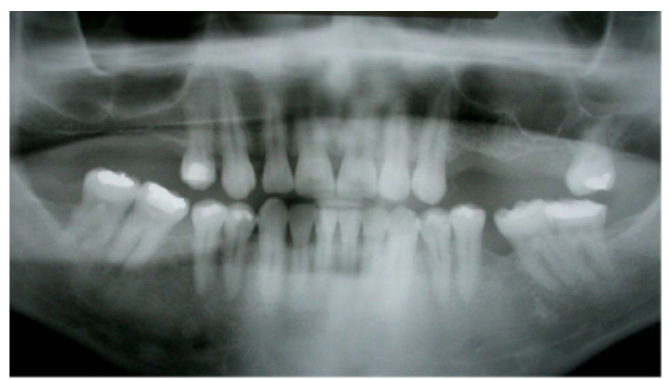

Fig. (1). Pre-operative OPG.

\subsection{Surgical Procedures}

Local anesthesia was administered in the buccal and palatal regions of the surgical area. For the lateral window approach, access to the lateral maxillary wall was achieved via a mucosal crestal incision and anterior and posterior releasing vertical incisions. A bony window was elevated with a round diamond bur on the lateral wall of the maxillary sinus. The window was then carefully removed. After dissection and elevation of the sinus membrane, bovine bone mineral (Bio-Oss; Geistlich) was used to fill the sub-membranous space. Bio-Oss is produced by Osteohealth Co. (Shirley, NY 11967) (Fig. 2). The bony window was replaced and closed with monofilament sutures. 
The implants were placed after a period of up to 8-9 months to allow for graft maturation (Figs. 3 \& 4). A standard OSFE technique was initiated for the other side of the posterior maxilla for the same patients. A mid-crestal incision was performed for flap elevation; vertical and periosteal release incisions were avoided. Cortical bone marking, for site positioning, was performed with 3 round burs of increasing diameters from 1.4 to $3.1 \mathrm{~mm}$. A $2.8 \mathrm{~mm}$-diameter sinus osteotome (Straumann AG, Basel, Switzerland) was performed to push the sinus floor axially. The use of osteotomes, rather than drills, prevented ovalization of the implant bed in the limited residual bone. The sinus floor was then broken by light strokes using a mallet and carefully pushed into the sinus cavity to a maximal height of $3 \mathrm{~mm}$; the Schneiderian membrane was further elevated by implant placement. The osteotomy site was enlarged by the 3.5 to $4.2 \mathrm{~mm}$-diameter sinus osteotome, based on the implant placement in the prepared osteotomy sites. The integrity of the membrane was controlled with an undersized depth gauge of $2.1 \mathrm{~mm}$. No grafting material was used in any of the cases. Implant insertion was performed without tapping. All of the patients underwent open-flap procedures for implant placement. ITI SLA solid screws (Straumann Ch Company of Basel, Switzerland manufacturer), 4.8 or $4.1 \mathrm{~mm}$ in diameter, were used for both sides. The flaps were sutured, and all of the implants achieved primary stability. The healing period was lengthy but uneventful. After surgery, the patients were asked to sit up comfortably in the dental chair and to rest for 30 minutes. The patients who were experiencing vertigo were asked to rest and sit up comfortably in the dental chair for an additional 15 to 30 minutes prior to discharge from the clinic to make sure that the patients are not experiencing vertigo after this time. Multiple Valsalva tests were performed in all of the cases to check for patency of the Schneiderian membrane immediately following the procedure. The patients were also instructed not to wear their dentures for 2 weeks post-operatively. Antibiotics (amoxicillin $500 \mathrm{mg}$ every 8 hours) were prescribed for 7 days, analgesics were given as needed, along with an antiseptic rinse with $0.2 \%$ chlorhexidine twice daily. The sutures were removed 7-10 days after surgery. Post-operative investigation records included X-rays (Figs. 5 \& 6), intraoral photographs, and survival rate chart of all implants for up to 24 months.

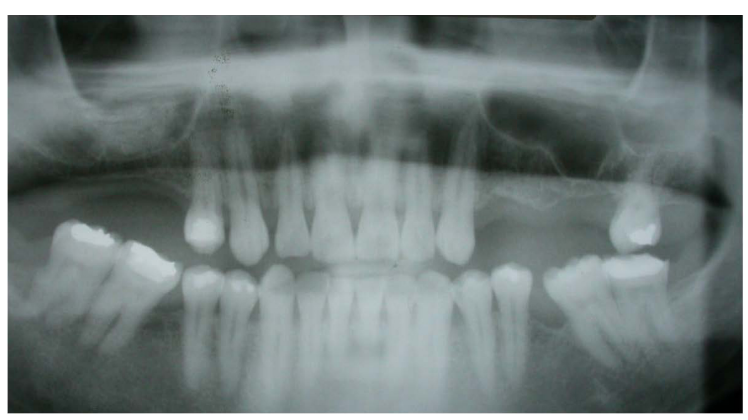

Fig. (2). Post-op-lateral approach with Bio-Oss graft on the right side.

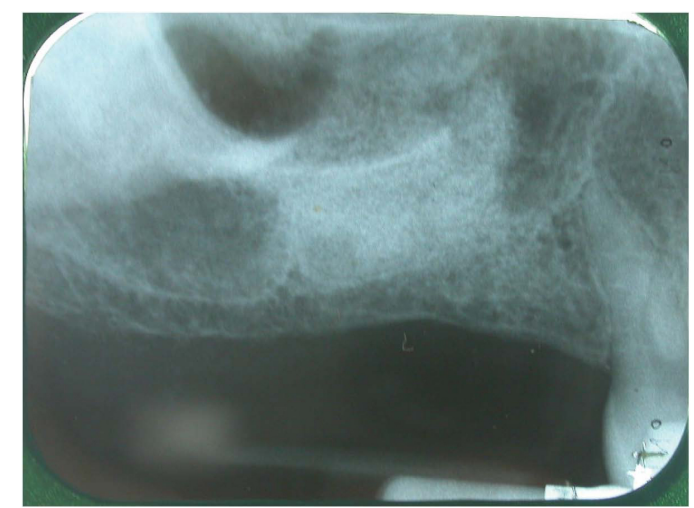

Fig. (3). Post-op lateral approach with healing phase on the right side.

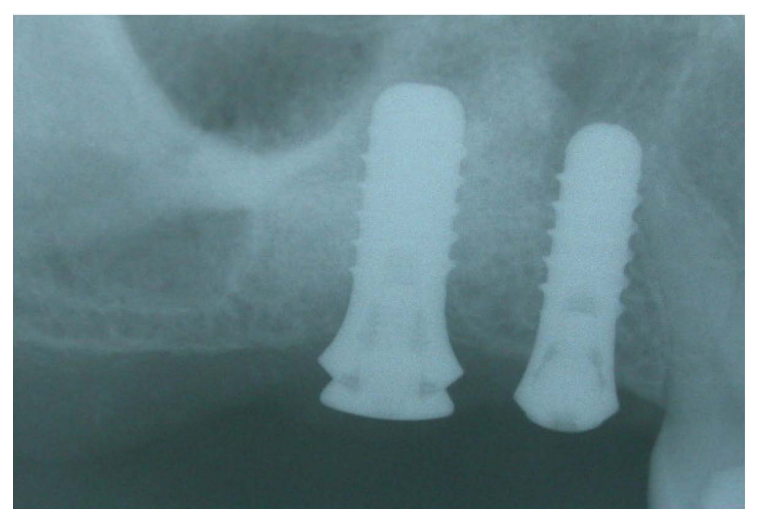

Fig. (4). Implant placement after undergoing the lateral approach on the right side.

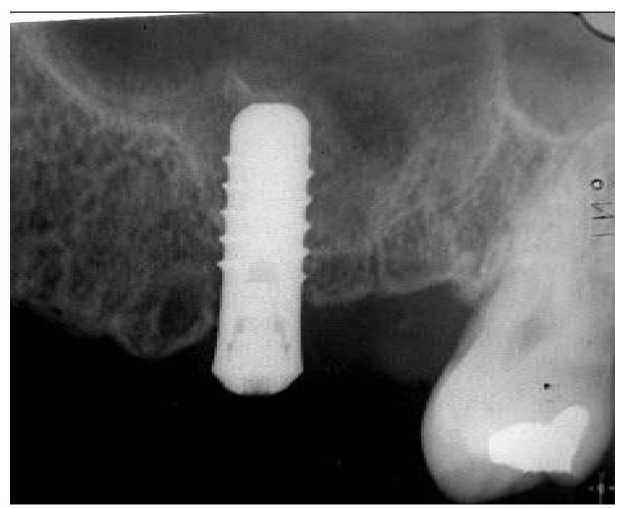

Fig. (5). Post-op crestal approach on the left side.

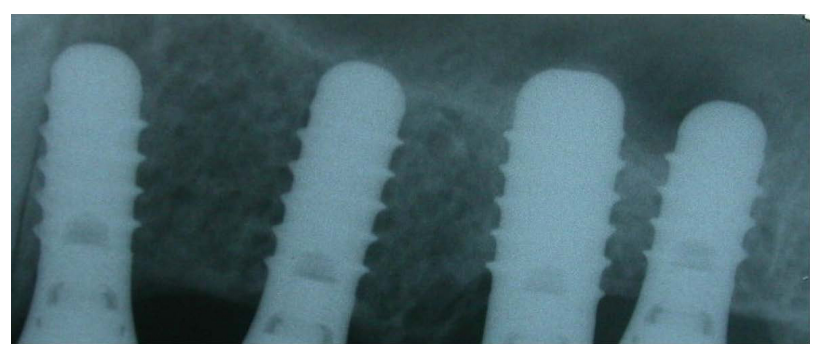

Fig. (6). Six months post-op after the crestal approach on the left side; the implant distal to the left canine did not interfere with the sinus lift or the distal side of the canine. 
Table 1. Distributions of implants placed in 10 patients and operation times.

\begin{tabular}{|c|c|c|c|c|c|c|}
\hline \multirow{2}{*}{ Patient Number } & \multicolumn{2}{|c|}{ Lateral Window Technique } & \multicolumn{2}{|c|}{ Osteotome Technique } & \multirow{2}{*}{ Total } & \multirow{2}{*}{ Preferred Approach } \\
\hline & No. of Implants & "Operation Time (min.) & No. of Implants & Operation Time (min.) & & \\
\hline Patient \# 1 & 2 & 75 & 3 & 45 & 5 & Did not reveal any preference \\
\hline Patient \# 2 & 2 & 70 & 1 & 31.5 & 3 & Crestal \\
\hline Patient \# 3 & 1 & 68 & 1 & 30.5 & 2 & Crestal \\
\hline Patient \# 4 & 2 & 80 & 2 & 33 & 4 & Crestal \\
\hline Patient \# 5 & 1 & 63 & 1 & 25.5 & 2 & Crestal \\
\hline Patient \# 6 & 2 & 84 & 2 & 34 & 4 & Crestal \\
\hline Patient \# 7 & 2 & 78 & 1 & 24 & 3 & Crestal \\
\hline Patient \# 8 & 1 & 67 & 1 & 27 & 2 & Crestal \\
\hline Patient \# 9 & 2 & 84 & 1 & 26 & 3 & Did not reveal any preference \\
\hline Patient \# 10 & 1 & 72 & 2 & 30 & 3 & Crestal \\
\hline Total & 16 & - & 15 & - & 31 & - \\
\hline- & - & Average $=74.1$ & - & Average $=30.65$ & - & $\begin{array}{l}\text { Differences } \\
43.45 \mathrm{~min} .\end{array}$ \\
\hline- & - & $\mathrm{SD}=7.294$ & - & $\mathrm{SD}=6.05$ & - & 1.244 \\
\hline
\end{tabular}

Average difference in operation time between the two techniques: $43.45 \mathrm{~min}$, SD: 1.244 . ${ }^{*}$ Operation time for the lateral approach is the total time for two stages ( $1^{\text {st }}$ stage: sinus lift with Bio-Oss graft and $2^{\text {nd }}$ stage: surgical implant placement).

Impressions were taken after a healing period of 6-9 months after implants insertion. Prosthetic abutments were inserted with $35 \mathrm{Ncm}$ of torque, and restorations were delivered (Figs. $7 \&$ 8). A Computed Tomography (CT) scans were obtained 48 months after loading the last implant.

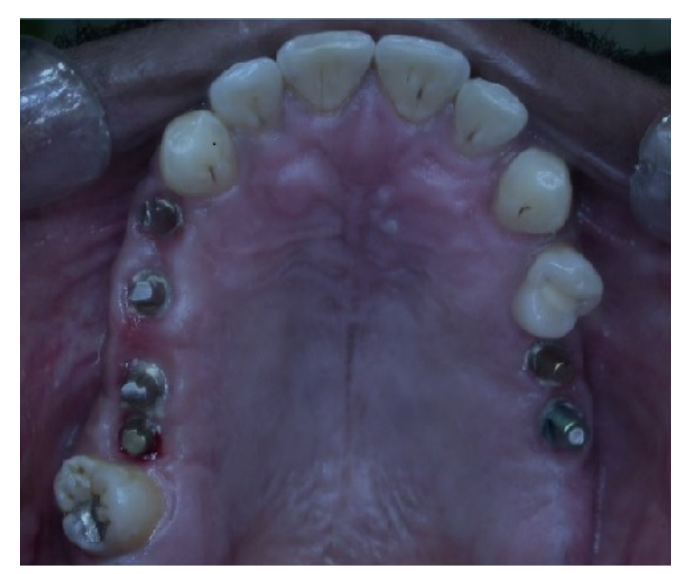

Fig. (7). Abutments in situ for both sides.

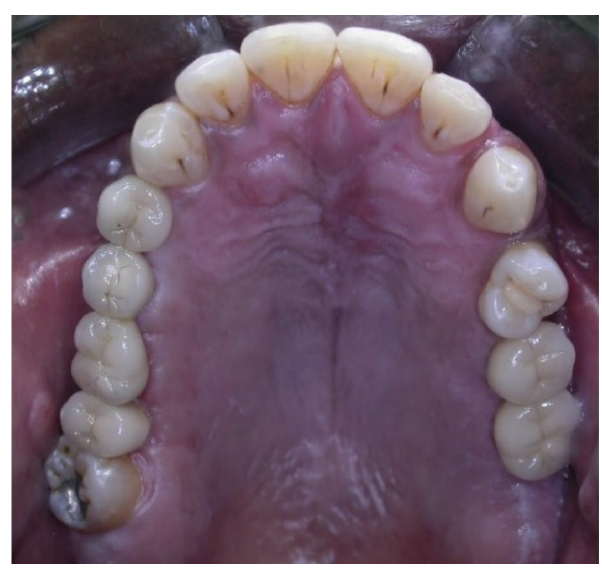

Fig. (8). Final prostheses for both sides.

\subsection{Patients' Perceptions}

After the surgical procedures, all of the patients were asked to provide their feedback on the surgical procedures and implant therapy. The patients were asked 14 questions and instructed to evaluate, criticize and compare both surgical approaches to determine subjective perceptions of maxillary sinus lift post-surgery expectations using a VAS scale. The VAS score of " 0 " indicates "total acceptance or no inconvenience' and " 10 " indicates 'total refusal, unpleasant or painful feelings' [19]. The average VAS score for each question was calculated and analyzed using the paired t-test to provide corresponding $p$-values. The timeline for each surgical procedure was also recorded (Table $\mathbf{1}$ ).

\subsection{Clinical Examinations}

At the annual clinical examination after functional loading, the survival of the implants and reconstructions were evaluated using the following clinical parameters:

- $\quad$ Pocket Probing Depth (PPD) in millimeters measured at four sites per implant;

- Distance between the implant shoulder and the mucosal margin (DIM), measured at all four sites of implantation (recession scored as a negative value); and

- Distance between the implant shoulder and first visible bone-implant contact (DIB).

All of the soft tissue measurements (made to the nearest millimeter), were performed using a UNC-15 periodontal probe (Hu-Friedy, Chicago, IL, USA).

\subsection{Radiographic Examinations}

All patients were recalled and programmed after the sinus lift procedure, implantation and prosthetic rehabilitation for orthopantomographic and intraoral radiographs, every 6 months for the first year. The radiographs were utilized to 
measure DIM and DIB. CT scans were obtained 48 months after loading the last implant.

The analysis of the data was performed using SPSS software, version 20.0. Unpaired t-tests were applied to compare the mean PPD, DIM and DIB between the bilateral sinus lift implant techniques with the $p$-value $\leq 0.05$ was considered significant.

\section{RESULTS}

All of the patients underwent similar procedures, with successful single attempts for both techniques (Figs. 9 \& 10). Note that case number 3: one of the implants at the osteotome side was not included, because it was not in the lifted sinus area). In the lateral window approach, implants were placed during second intervention. Out of 31 ITI solid screw implants $4.1 \mathrm{~mm}$ and $4.8 \mathrm{~mm}$ in diameter, 15 implants were utilized for OSFE, and 16 implants were used for the lateral approach. The implant lengths used were $10 \mathrm{~mm}$ and $12 \mathrm{~mm}$ in both techniques. At the time of abutment connection, all of the implants were stable. The mean follow-up period was 36 months after permanent prosthetic insertion. During follow-up period, all implants and prostheses were stable and functional, no pain or swelling were noted in any of the cases before or after prosthetic loading.

\subsection{Survival Failure Rates}

There were no complications associated with the surgical procedure; such as infection of the maxillary sinus, loss of bone particles through the nose, wound dehiscence, and/or loss of the implants' initial stability. One sinus membrane perforation was noted during the lateral window approach but was not considered a complication related to the surgical procedures as it was a minimal perforation noted at the initial bur hole region. The perforation was managed by leaving the membrane folded over itself while lifting the sinus membrane, thus obviating the need for repair. All of the implants presented had radiologically proven osseointegration and thus received single crowns or fixed prostheses. All of the implants successfully fulfilled the Buser et al. criteria [18].

\subsection{Radiographic Assessments}

Linear measurements were obtained to evaluate the marginal bone levels. The DIB was measured. None of the periapical radiographs revealed any peri-implant radiolucency during the follow-up period. However, minimal crestal bone loss was noticed six to nine months after loading. There were no significant differences in the marginal bone levels between the lateral window and the OSFE implants. Radiographically, the apical elevation of the sinus floor for both approaches was observed. There was predictable radiography bones/graft established around the implant apex in cases completed with the lateral approach. There were no obvious confirmations seen of bone formation between the lifted sinus membrane and the implant apex.

CT scans showed the amount of bone gained bilaterally by both techniques, as shown on right and left sagittal CT films (Fig. 11) and coronal CT scans (Fig. 12). The reformatted flythrough image of the maxillary sinus floor (Figs. $13 \& 14$ ) showed an intact Schneiderian membrane over the projection of the apical border of the implant at both sides. After two years of prosthetic loading, periapical radiographs showed a stable clinical situation in the area around the apices of the implants on both sides. A dome-shaped structure was apparent at the sites of the first and second molars on the left side (Fig. 15). Selected magnified images from CT scans showed the apical borders for these implants, the surrounding bone, and the new sinus floor 2 years after surgery using the staged OSFE technique on the left side (Fig. 16). No peri-implant radiolucency was distinguished in any of the cases.

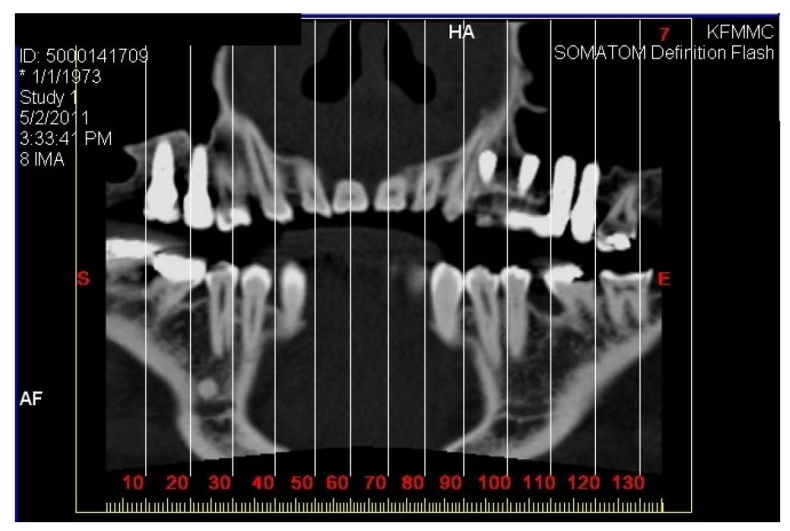

Fig. (9). CT scan showing uniformity of bone formation.

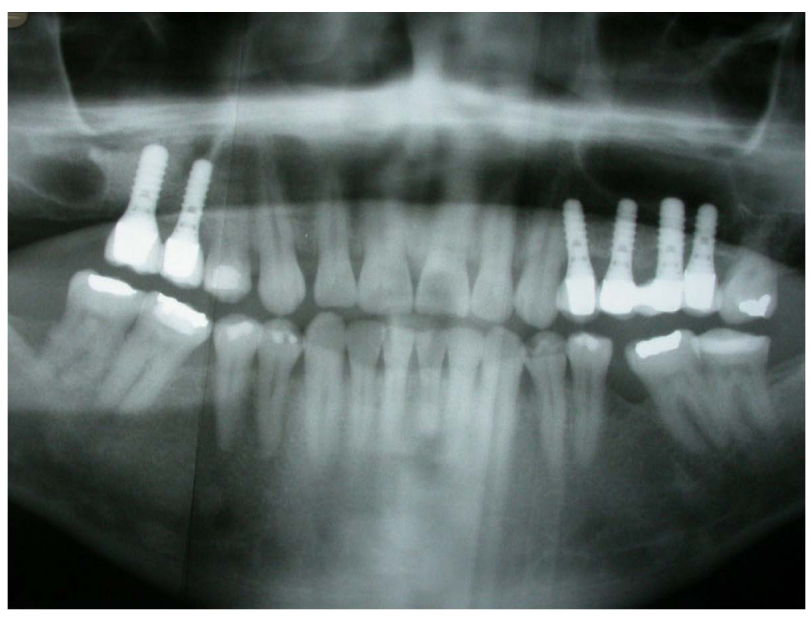

Fig. (10). OPG compares bone formation on both sides.
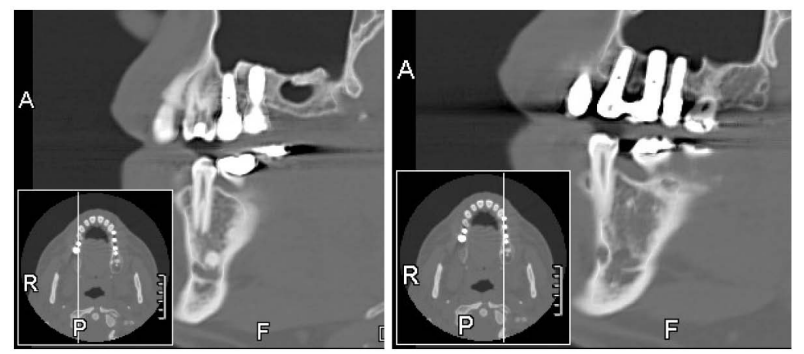

Fig. (11). Sagittal CT showing bone formation on both sides. 


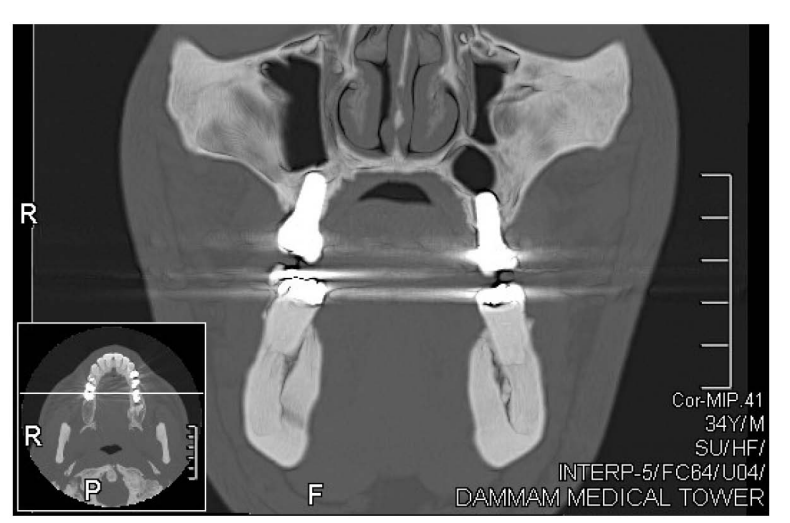

Fig. (12). Coronal CT showing bilateral bone formation.

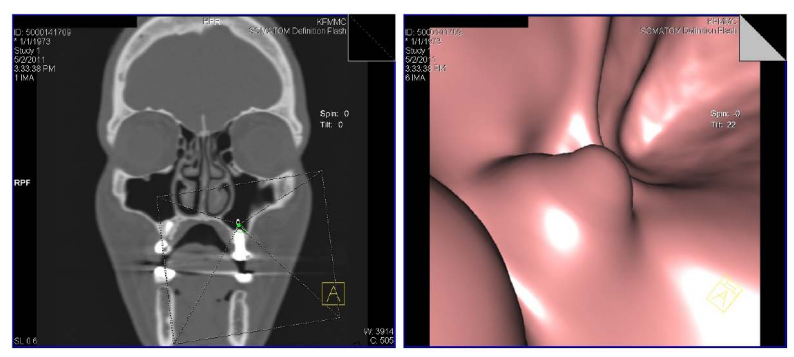

Fig. (13). Syngo Siemens Software was used for the thin cut images in the navigation protocol-Fly_through Application-left side.
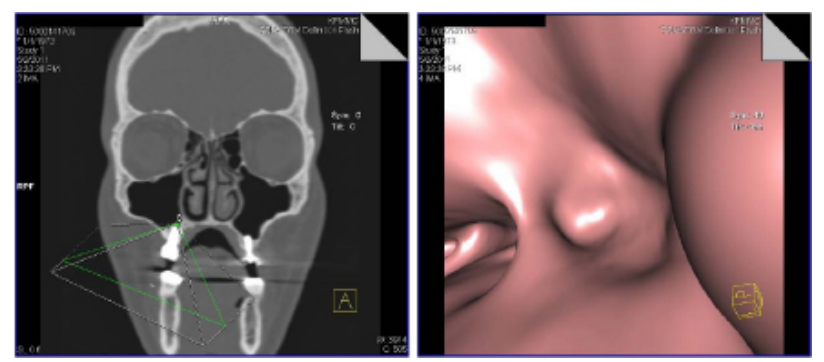

Fig. (14). Syngo Siemens Software was used for the thin cut images in the navigation protocol-Fly_through Application-right side.

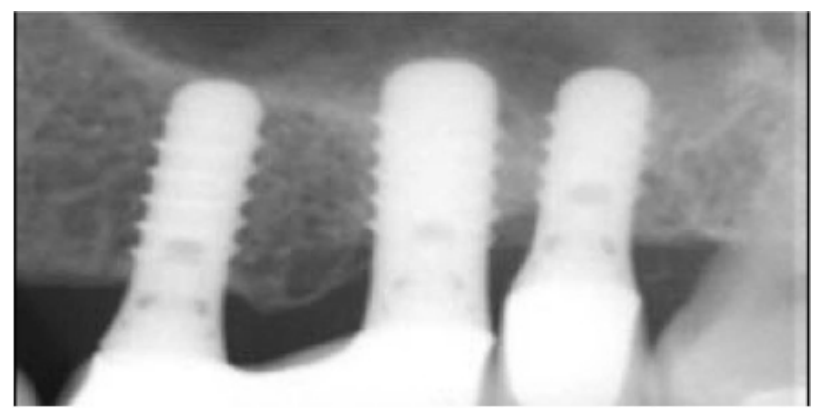

Fig. (15). Periapical radiograph after two years of prosthetic loading. A dome-shaped structure was apparent at the sites of the first and second molars.

\subsection{Peri-Implant Soft Tissue Condition}

Using 2-tailed independent samples t-tests, there were no significant differences between the two approaches (lateral and osteotome) in the averages of 3 indices (PPD, DIM and DIB). The $p$-values of the tests for PPD, DIM and DIB were 0.0504, 0.7784 and 0.1817 , respectively. The proximities of these averages in the 3 indices comparing the two approaches to the boundaries are shown in Table $\mathbf{2}$. The mean and standard deviation for the marginal bone level of the lateral window implants was $2.67 \pm 0.95 \mathrm{~mm}$, compared with $2.75 \pm 0.95 \mathrm{~mm}$ for the OSFE implants. The mean and standard deviation of PPD for the implants with the lateral window approach was $3.83 \pm 0.348 \mathrm{~mm}$, compared with $3.55 \pm 0.406 \mathrm{~mm}$ for implants using the crestal approach. The mean and standard deviation for DIM for implants with the lateral window approach was $0.356 \pm 0.784 \mathrm{~mm}$, compared with $0.447 \pm 0.613$ $\mathrm{mm}$ for implants with the crestal approach. The mean and standard deviation for DIB for implants with the lateral window approach was $2.85 \pm 0.465 \mathrm{~mm}$, compared with $2.64 \pm$ $0.397 \mathrm{~mm}$ for implants with the crestal approach (Table 2).
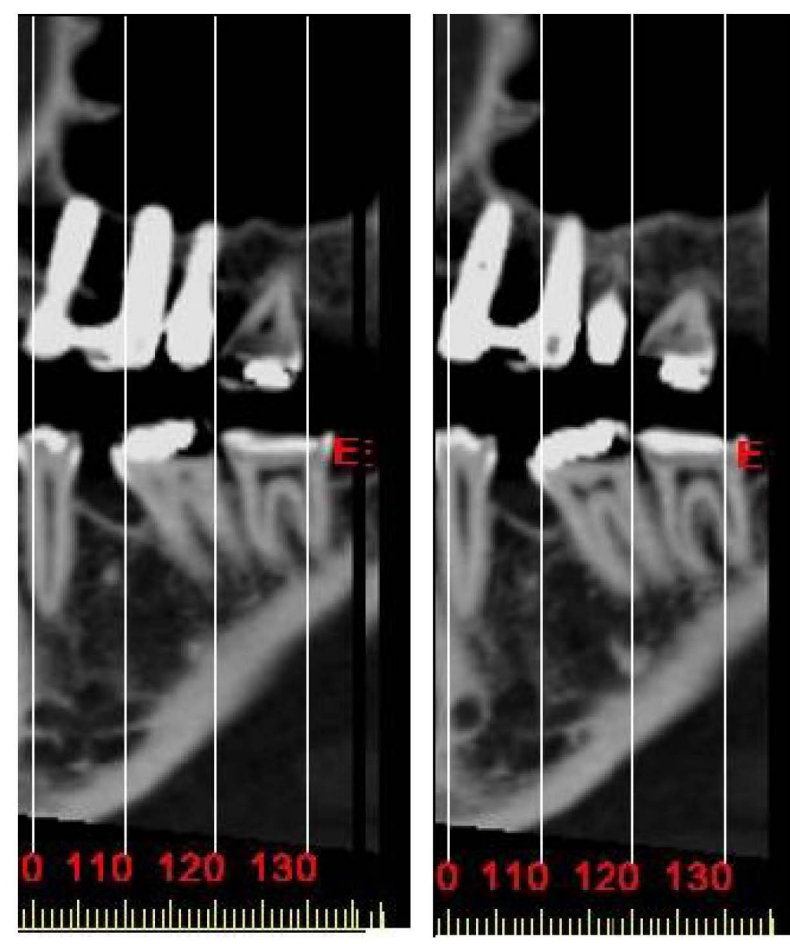

Fig. (16). Selected magnified images of CT scans showing the apical borders for these implants, the surrounding bone, and the new sinus floor using crestal technique on the left side, 36 months after the operation.

Statistically, there was no significant difference in the mean mesial and distal PPD between the lateral and osteotome bilateral sinus lift implant techniques. Additionally, there was no significant difference in the mean DIM for the buccal, lingual, mesial or distal aspects between the two sinus lift implant techniques $(p>0.05)$ and the mean mesial and distal DIB between the lateral and osteotome bilateral sinus lift implants $(p>0.05)$. Finally, there were no significant differences in the average PPD, DIM, and DIB between the lateral and crestal approaches (Table 2 and Fig. 17). 
Table 2. Mean comparison of PPD, DIM and DIB between the lateral and osteotome methods.

\begin{tabular}{|c|c|c|c|}
\hline Variables & $\begin{array}{l}\text { Lateral } \\
(\mathrm{n}=16)\end{array}$ & $\begin{array}{c}\text { Osteotome } \\
(\mathrm{n}=15)\end{array}$ & $P$-Value \\
\hline \multicolumn{4}{|c|}{ Pocket Probing Depth } \\
\hline - Buccal & $3.78 \pm 0.329$ & $3.55 \pm 0.421$ & 0.099 \\
\hline - Lingual & $3.78 \pm 0.368$ & $3.41 \pm 0.531$ & 0.031 \\
\hline - Mesial & $3.92 \pm 0.415$ & $3.64 \pm 0.511$ & 0.103 \\
\hline - Distal & $3.81 \pm 0.398$ & $3.59 \pm 0.398$ & 0.130 \\
\hline - Average & $3.83 \pm 0.348$ & $3.55 \pm 0.406$ & 0.0504 \\
\hline \multicolumn{4}{|c|}{ Distance between implant mucosal margins } \\
\hline - Buccal & $-0.356 \pm 0.666$ & $-0.447 \pm 0.596$ & 0.694 \\
\hline - Lingual & $-0.381 \pm 0.779$ & $-0.360 \pm 0.722$ & 0.938 \\
\hline - Mesial & $-0.356 \pm 0.784$ & $-0.447 \pm 0.613$ & 0.724 \\
\hline - Distal & $-0.356 \pm 0.764$ & $-0.467 \pm 0.595$ & 0.658 \\
\hline - Average & $-0.363 \pm 0.722$ & $-0.431 \pm 0.594$ & 0.777 \\
\hline \multicolumn{4}{|c|}{ Distance between the implant shoulder and first visible bone-implant contact } \\
\hline - Mesial & $2.88 \pm 0.536$ & $2.56 \pm 0.484$ & 0.084 \\
\hline - Distal & $2.81 \pm 0.444$ & $2.71 \pm 0.456$ & 0.552 \\
\hline - Average & $2.85 \pm 0.465$ & $2.64 \pm 0.397$ & 0.184 \\
\hline
\end{tabular}

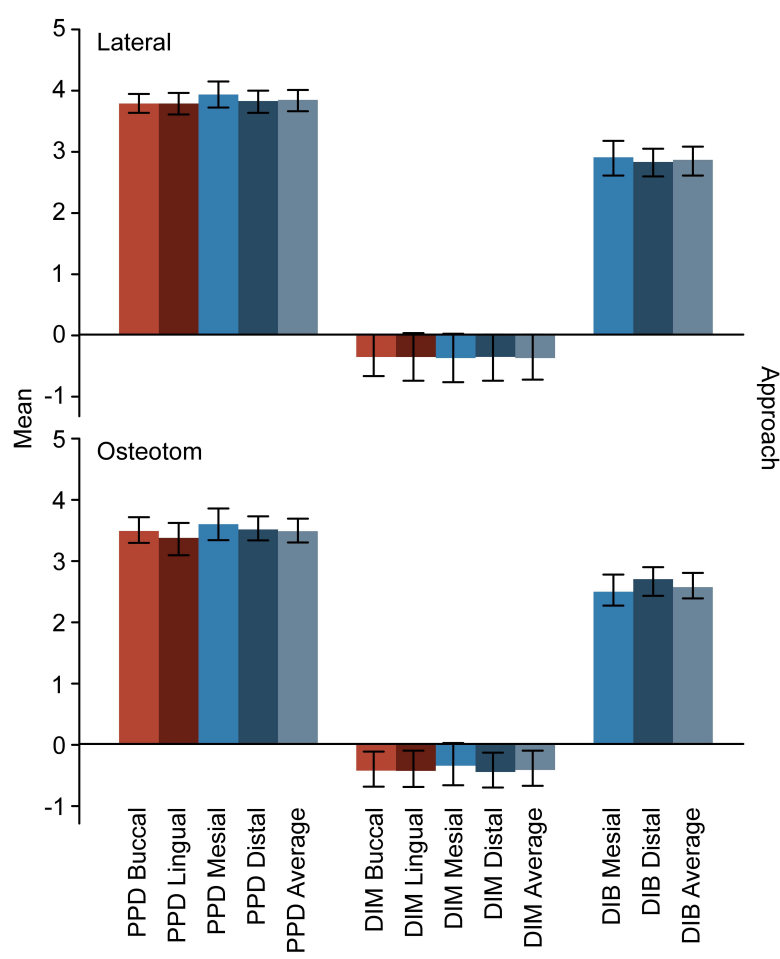

Fig. (17). There were non-significant differences in the average PPD, DIM and DIB between the lateral and osteotome approaches.

\subsection{Patient-Centered Outcomes}

All 10 patients actively participated in evaluating, criticizing and comparing both surgical procedures which included general satisfaction, nausea, vertigo, pain and discomfort after the surgical procedures. Using the VAS scale, with " 0 " to indicate 'total acceptance or no inconvenience' and " 10 " to indicate 'total refusal or unpleasant or painful feelings.' Conce- rning general satisfaction, the questionnaire consisted of 14 questions concerning the two approaches. All of the patients had receptive attitudes toward the questionnaire, and all ten patients answered all of the questions about both approaches. The provided mean and standard deviation for the VAS score for all of the questions related to the lateral approach was 2.92 \pm 1.050 , compared to $3.2 \pm 0.9751$ for the crestal approach $(P \geq$ $0.05)$. The analysis demonstrated no statistically significant difference in the total VAS scores between the lateral and crestal approaches (Table 3 and Fig. 18). The timeline of the study for both crestal and lateral approaches within 36 months was shown in (Fig. 19), which demonstrated the implant placement for lateral approach 8-9 months after sinus floor elevation with a Bio-Oss graft. None of our patients required any medications for such complaints. In the overall assessment, eight of the ten patients preferred the OSFE over the lateral approach with the remaining patients not revealing any preference (Table 1).

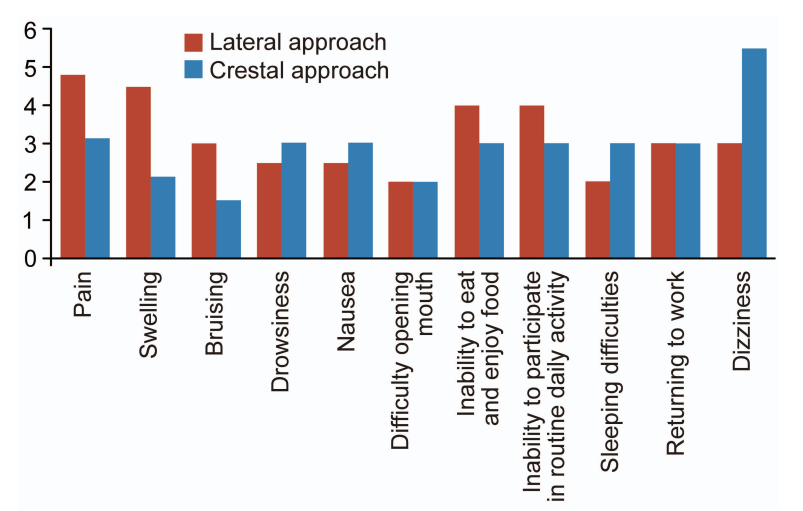

Fig. (18). Determination of subjective maxillary sinus lift post-surgery expectations using a VAS. There were non-significant differences in the questionnaire for all symptoms and patients on the total VAS scale between crestal and lateral approaches. 
Table 3. Determination of subjective maxillary sinus lift post-surgical expectations using a VAS, in which 0 indicated "total acceptance or no inconvenience" and 10 indicated "total refusal or unpleasant or painful feeling".

\begin{tabular}{|c|c|c|c|}
\hline $\begin{array}{c}\text { Average VAS Scale for each Question } \\
\text { Lateral Approach }\end{array}$ & $\begin{array}{c}\text { Average VAS Scale for each Question } \\
\text { Crestal Approach }\end{array}$ & Questionnaire for each Symptom Score from 0 to 10 & - \\
\hline 4.8 & 3.1 & Pain & 1 \\
\hline 4.5 & 2.1 & Swelling & 2 \\
\hline 3 & 1.5 & Bruising & 3 \\
\hline Negligible (0) & Negligible (0) & Nasal bleeding & 4 \\
\hline 2.5 & 3 & Drowsiness & 5 \\
\hline 2.5 & 3 & Nausea & 6 \\
\hline 2 (highest level on $1^{\text {st }}$ day) & 2 (highest level on $1^{\text {st }}$ day) & Difficulty with mouth opening & 7 \\
\hline 4 (highest level on $1^{\text {st }}$ to $3^{\text {rd }}$ days) & 3 (highest level on $1^{\text {st }}$ to $3^{\text {rd }}$ days) & Inability to eat and enjoy food & 8 \\
\hline 4 (highest level on $1^{\text {st }}$ to $3^{\text {rd }}$ days) & 3 (highest level on $1^{\text {st }}$ to $3^{\text {rd }}$ days) & Inability to participate in routine daily activity & 9 \\
\hline 2 & 2 & Sleeping difficulties & 10 \\
\hline 3 (sick leave for 3 days) & 3 (sick leave for 3 days) & Returning to work & 11 \\
\hline 3 & $\begin{array}{l}\text { 5.5. immediately after surgery, lasted a } \\
\text { maximum of } 30 \mathrm{~min} \text {. }\end{array}$ & Dizziness (vertigo) & 12 \\
\hline Negligible (0) & Negligible (0) & Difficulty in nasal airflow at the sinus lift area & 13 \\
\hline Negligible (0) & Negligible $(0)$ & Breathing, swallowing, and speech difficulties & 14 \\
\hline Average $=3.2$ & Average $=2.92$ & - & - \\
\hline $\mathrm{SD}=0.9751$ & $\mathrm{SD}=1.050$ & $P$-value $=2.22$ & - \\
\hline
\end{tabular}

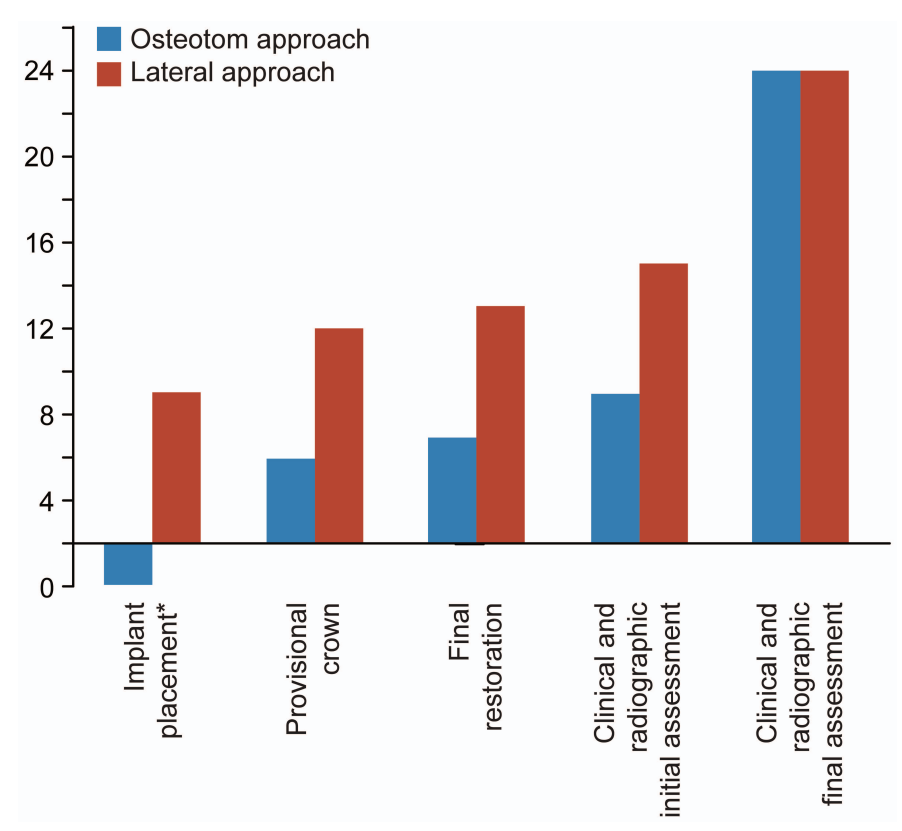

Fig. (19). Timeline of the study for both crestal and lateral approaches within 36 months. *Implant placement for the lateral approach $8-9$ months after sinus floor elevation with a Bio-Oss graft.

\section{DISCUSSION}

This article is the full version of a previous short communication published earlier [20]. Being this was a splitmouth study, the comparison of both techniques in the same patients proved that the symptoms seen only in the OSFE group may be specific to the technique. The crossover effect of learned memory in the split-mouth design is one of the limitations of this study [14]. Checchi et al. conducted a randomized clinical trial with a split-mouth design to compare the Summers and Cosci techniques in crestally augmented sinuses with particulate cancellous human allografts [17]. To reduce the crossover effect, all of the patients were educated regarding the procedural steps, and they provided informed consent for both techniques prior to the first procedure. The crossover effect might have been further reduced in this study because six of the ten cases had crestal approaches followed by OSFE, whereas remaining four patients had OSFE procedures before the crestal approach. Patients who experienced OSFEassociated symptoms during and after the OSFE did not experience any of these symptoms with the lateral approach. 
However, all of the patients recovered from these symptoms after 30 to 60 minutes. It was interesting to note that based on their responses, none of the patients were concerned about this transient discomfort.

Average difference in operation time between the two techniques: 43.45 , SD: 8.254 . ${ }^{*}$ Operation time for the lateral approach is the total time for two stages $\left(1^{\text {st }}\right.$ stage: sinus lift with Bio-Oss graft and $2^{\text {nd }}$ stage: surgical implant placement).

The symptoms of vertigo can require pharmacological management to reduce the spinning sensations and/or to reduce the accompanying nausea. The most commonly used drugs are anxiolytics, sedatives, and/or muscle relaxants, along with antihistamines. Antihistamines appear to have suppressive effects on the central emetic center, relieving nausea and vomiting associated with motion sickness [21]. None of our patients required such medications. To prevent this complication, a gentle hammering should be performed, and a careful approach should be taken during the osteotome technique. Patients should be informed regarding the possibility of postoperative vestibular symptoms, because these symptoms can be very unpleasant and may cause considerable stress if the patient was not previously informed of this problem. Although not indicated in this study, if the symptoms are incapacitating, immediate referral to an otorhinolaryngologist is recommended [22 - 26].

There was no significant difference in the total VAS scale between the crestal and lateral approaches; $P=2.22$.

The time required for implant placement after the lateral approach was mentioned negatively in the responses of all of the patients to the questionnaire. This finding might have been influenced by the current expectations of patients in this era of immediate loading implants. Although a delay in implant placement was the main reason for preferring OSFE over the lateral approach, the lateral approach was the choice of patients who feared the development of such symptoms and of patients who could not tolerate the discomfort of hammering. This finding also emphasizes the importance of disclosing all vestibular symptoms when presenting the OSFE procedure to the patient.

Different techniques have attempted to reduce the force exerted during the fracture of the nasal floor in OSFE. Romanos described a technique for window preparation for the sinus lift procedure [27]. A round bur was used to prepare the osteotomy under continuous saline solution irrigation. Various techniques are now being used to reduce the membrane perforation, including the use of piezosurgery, hydrostatic force, laser surgery, various modifications of hammer osteotomes, and more precise radiological evaluation during surgery. All of these techniques could also reduce the perforation rate in OSFE [28 - 37].

Radiological evaluation revealed sufficient lifting of the sinus floor by both techniques. Radiological evidence of the presence of bone over the implant apex was proved by CT scans for all of the patients. Radiological evidence of bone formation at the apex of the implant on periapical radiographs was not always confirmed in the OSFE cases, whereas with the lateral technique, sufficient bone could be observed above the implant apex. Nevertheless, there has been no inconclusive clinical evidence to prove any advantage of bone over the implant apex directly affecting implant survival in any of the sinus lifting procedures [38, 39].

Although various biomaterials are currently being used to augment beneath the lifted sinus membrane in both the OSFE and lateral techniques, biomaterials were not utilized after OSFE in this study. Piero et al. assessed the clinical outcomes of sinus membrane elevation in combination with implant placement without any biomaterials [40]. Although the regeneration of bone was not uniform, the height of the membrane lift did not correlate with the amount of regenerated bone, and the success rate was not affected. However, with the lateral approach without simultaneous implant placement, grafting material is often necessary to support the sinus membrane [41 - 44]. Membrane perforations were easily visualized with the lateral approach compared to the OSFE, whereas with OSFE, other clinical parameters, including the Valsalva test, were utilized to confirm membrane integrity. Some authors believe that tearing of the Schneiderian membrane was a factor that diminished implant survival rate [45]. Hernandez-Alfaro et al. observed an implant survival rate that was inversely proportional to the size of the perforation [46]. However, some authors did not report tearing of the membrane to be a negative factor in the survival rates of implants [47]. Schwartz-Arad et al. reported that tearing of the membrane influenced the occurrence of post-surgical complications but did not influence the survival rate of the implants [48]. Membrane integrity has considerable significance in the lateral approach, because it limits the amount of sinus graft material inserted into the zone, improving implant survival and reducing complications. Ardekian et al. did not find significant differences in implant survival rates between implants inserted in a grafted sinus with a perforated membrane and in a sinus with an intact membrane [47]. The criteria used to select the lateral technique over OSFE were the residual bone height and the predicted initial stability, rather than avoidance of membrane perforation by direct visualization $[49,50]$. One of the ten cases in this study had a membrane perforation during the lateral approach. However symptoms such as nasal bleeding, graft escape or nasal congestion were not reported in this case.

Radiological data were used to assess implant success. The Periotest apparatus has been shown to be successful in assessing the stability of implants. However, it often fails to detect cases with saucerization of the bone. Radiographs can show the bone levels of implants. It was found that, although an implant was stable, it might have suffered significant horizontal bone loss $[51,52]$. The quality and quantity of the bone covering the implant apex might prevent the effects of tangential forces applied on loaded implants, which could eventually initiate crestal bone loss. In OSFE, because there is no marked bone formation around the implant apex, these tangential forces can apply more rotational force, with a fulcrum situated toward the crestal bone, compared to an implant inserted using the lateral approach, in which a bone graft is used [38, 39]. The distinguishing of the bone created when replacing the bone graft with the lateral approach was different from the bone created around the implant with the OSFE approach. However, such a comparison was beyond the 
scope of the study. Nevertheless, there were no significant differences noted in the measurements of PPD, DIB or DIM between the two techniques. No implants were lost, and no significant differences were detected among the studied variables, and no or reduced crestal bone resorption was seen in one-year post-operative radiographs; therefore, the procedures were considered to have attained implant success, per Buser et al.'s criteria [19]. This was a split-mouth study that challenged a meticulous and direct assessment of both techniques for maxillary sinus lift. A split-mouth design with a larger sample size, directly comparing OSFE to the lateral approach with immediate implantation, could be considered in future studies.

\section{CONCLUSION}

Maxillary sinus floor elevation with both the crestal and OSFE techniques could be evaluated precisely when both techniques were performed in the same patient. Although the small sample size for a split-mouth study was a limitation, the specific advantages, disadvantages and indications for each technique could be distinguished. No significant differences were observed in PPD, DIM, and DIB when assessing implant survival using clinical and radiological assessments. Approximately $33.3 \%$ of the patients experienced vestibular discomfort during the OSFE procedures; however, no significant differences were noted in implant success between the two approaches. Approximately $80 \%$ of the patients preferred the OSFE approach over the lateral approach due to the delay in implant placement with the lateral approach.

\section{ETHICS APPROVAL AND CONSENT TO PARTI- CIPATE}

The Ethics Committee of the Medical Association for the Military Hospital, Saudi Arabia approved the study for publication. (Research Ethics Committee REC Number: kfmmc-dd18/05/2011)

\section{HUMAN AND ANIMAL RIGHTS}

No animals were used in this research. All research procedures on humans were followed in accordance with the ethical standards of the committee responsible for human experimentation (institutional and national), and with the Helsinki Declaration of 1975, as revised in 2013. (http://ethics.iit.edu/eco des/node/3931)

\section{CONSENT FOR PUBLICATION}

The participants provided written informed consent.

\section{AVAILABILITY OF DATA AND MATERIAL}

The authors confirm that the data supporting the findings of this study are available within the article.

\section{FUNDING}

None.

\section{CONFLICT OF INTEREST}

The authors declare no conflict of interest, financial or otherwise.

\section{ACKNOWLEDGEMENTS}

Declared none.

\section{REFERENCES}

[1] Misch CE. Book of Contemporary Implant Dentistry. $2^{\text {nd }}$ ed. St. Louis: Mosby 1999.

[2] Esposito M, Hirsch JM, Lekholm U, Thomsen P. Biological factors contributing to failures of osseointegrated oral implants. (II). Etiopathogenesis. Eur J Oral Sci 1998; 106(3): 721-64. [http://dx.doi.org/10.1046/j.0909-8836..t01-6-.x] [PMID: 9672097]

[3] Boyne PJ, James RA. Grafting of the maxillary sinus floor with autogenous marrow and bone. J Oral Surg 1980; 38(8): 613-6. [PMID: 6993637]

[4] Tatum H Jr. Maxillary and sinus implant reconstructions. Dent Clin North Am 1986; 30(2): 207-29. [PMID: 3516738]

[5] Hallman M, Sennerby L, Lundgren S. A clinical and histologic evaluation of implant integration in the posterior maxilla after sinus floor augmentation with autogenous bone, bovine hydroxyapatite, or a 20:80 mixture. Int J Oral Maxillofac Implants 2002; 17(5): 635-43. [PMID: 12381063]

[6] Strietzel FP, Nowak M, Küchler I, Friedmann A. Peri-implant alveolar bone loss with respect to bone quality after use of the osteotome technique: Results of a retrospective study. Clin Oral Implants Res 2002; 13(5): 508-13.

[http://dx.doi.org/10.1034/j.1600-0501.2002.130510.x] [PMID: 1245 3128]

[7] Jakse N, Seibert FJ, Lorenzoni M, Eskici A, Pertl C. A modified technique of harvesting tibial cancellous bone and its use for sinus grafting. Clin Oral Implants Res 2001; 12(5): 488-94.

[http://dx.doi.org/10.1034/j.1600-0501.2001.120509.x] [PMID: 1156 4109]

[8] Tadjoedin ES, de Lange GL, Lyaruu DM, Kuiper L, Burger EH. High concentrations of bioactive glass material (BioGran) $v s$. autogenous bone for sinus floor elevation. Clin Oral Implants Res 2002; 13(4): 428-36.

[http://dx.doi.org/10.1034/j.1600-0501.2002.130412.x] [PMID: 1217 5381]

[9] Summers RB. The osteotome technique Part 3: Less invasive methods of elevating the sinus floor. Compendium 1994; 15(6): 698-704. [PMID: 7994726]

[10] Cordioli G, Mazzocco C, Schepers E, Brugnolo E, Majzoub Z. Maxillary sinus floor augmentation using bioactive glass granules and autogenous bone with simultaneous implant placement. Clinical and histological findings. Clin Oral Implants Res 2001; 12(3): 270-8. [http://dx.doi.org/10.1034/j.1600-0501.2001.012003270.x] [PMID: 11359485]

[11] Manso MC, Wassal T. A 10-year longitudinal study of 160 implants simultaneously installed in severely atrophic posterior maxillas grafted with autogenous bone and a synthetic bioactive resorbable graft. Implant Dent 2010; 19(4): 351-60.

[http://dx.doi.org/10.1097/ID.0b013e3181e59d03] [PMID: 20683292]

[12] Checchi L, Felice P, Antonini ES, Cosci F, Pellegrino G, Esposito M. Crestal sinus lift for implant rehabilitation: A randomised clinical trial comparing the Cosci and the Summers techniques. A preliminary report on complications and patient preference. Eur J Oral Implantology 2010; 3(3): 221-32. [PMID: 20847992]

[13] Ferrigno N, Laureti M, Fanali S. Dental implants placement in conjunction with osteotome sinus floor elevation: A 12-year life-table analysis from a prospective study on 588 ITI implants. Clin Oral Implants Res 2006; 17(2): 194-205.

[http://dx.doi.org/10.1111/j.1600-0501.2005.01192.x] [PMID: 16584416]

[14] Lesaffre E, Philstrom B, Needleman I, Worthington H. The design and analysis of split-mouth studies: What statisticians and clinicians should know. Stat Med 2009; 28(28): 3470-82.

[http://dx.doi.org/10.1002/sim.3634] [PMID: 19536744]

[15] Donner A, Zou GY. Methods for the statistical analysis of binary data in split-mouth designs with baseline measurements. Stat Med 2007; 26(18): 3476-86.

[http://dx.doi.org/10.1002/sim.2782] [PMID: 17149800]

[16] Rotundo R, Nieri M, Cairo F, et al. Lack of adjunctive benefit of Er:YAG laser in nonsurgical periodontal treatment: A randomized split-mouth clinical trial J Clin periodont 2010; 37: 526-33. 
[17] Al-Almaie S, Kavarodi AM, Al Faidhi A. Maxillary sinus functions and complications with lateral window and osteotome sinus floor elevation procedures followed by dental implants placement: A retrospective study in 60 patients. J Contemp Dent Pract 2013; 14(3): 405-13.

[http://dx.doi.org/10.5005/jp-journals-10024-1336] [PMID: 24171981]

[18] Buser D, Weber HP, Brägger U. The treatment of partially edentulous patients with ITI hollow-screw implants: Presurgical evaluation and surgical procedures. Int J Oral Maxillofac Implants 1990; 5(2): 165-75.

[PMID: 2133341]

[19] Ahn SH, Park EJ, Kim ES. Reamer-mediated transalveolar sinus floor elevation without osteotome and simultaneous implant placement in the maxillary molar area: Clinical outcomes of 391 implants in 380 patients. Clin Oral Implants Res 2012; 23(7): 866-72.

[http://dx.doi.org/10.1111/j.1600-0501.2011.02216.x] [PMID: 2172 2189]

[20] Al-Almaie S, Kavarodi AM, Alorf A, Alzahrani S. A split-mouth design comparison for lateral and crestal sinus lift techniques with dental implants placements: Short communication. Open Dent J 2017; 11: 603-8.

[http://dx.doi.org/10.2174/1874210601711010603] [PMID: 29290838]

[21] Saker M, Ogle O. Benign paroxysmal positional vertigo subsequent to sinus lift via closed technique. J Oral Maxillofac Surg 2005; 63(9): 1385-7.

[http://dx.doi.org/10.1016/j.joms.2005.05.296] [PMID: 16122608]

[22] Kim MS, Lee JK, Chang BS, Um HS. Benign paroxysmal positional vertigo as a complication of sinus floor elevation. J Periodontal Implant Sci 2010; 40(2): 86-9.

[http://dx.doi.org/10.5051/jpis.2010.40.2.86] [PMID: 20498765]

[23] Sammartino G, Mariniello M, Scaravilli MS. Benign paroxysmal positional vertigo following closed sinus floor elevation procedure: Mallet osteotomes vs. screwable osteotomes. A triple blind randomized controlled trial. Clin Oral Implants Res 2011; 22(6): 669-72.

[http://dx.doi.org/10.1111/j.1600-0501.2010.01998.x] [PMID: 21054 553]

[24] Vernamonte S, Mauro V, Vernamonte S, Messina AM. An unusual complication of osteotome sinus floor elevation: Benign paroxysmal positional vertigo. Int J Oral Maxillofac Surg 2011; 40(2): 216-8. [http://dx.doi.org/10.1016/j.ijom.2010.07.010] [PMID: 20801616]

[25] Ramakrishna J, Goebel JA, Parnes LS. Efficacy and safety of bilateral posterior canal occlusion in patients with refractory benign paroxysmal positional vertigo: Case report series. Otol Neurotol 2012; 33(4): 640-2.

[http://dx.doi.org/10.1097/MAO.0b013e31824bae56] [PMID: 2242 9946]

[26] Reddy K S, Shivu ME, Billimaga A. Benign paroxysmal positional vertigo during lateral window sinus lift procedure: A case report and review. Implant Dent 2015; 24(1): 106-9.

[http://dx.doi.org/10.1097/ID.0000000000000188] [PMID: 25621557]

[27] Romanos GE. Window preparation for sinus lift procedures: A simplified technique. Implant Dent 2008; 17(4): 377-81. [http://dx.doi.org/10.1097/ID.0b013e31818c65f6] [PMID: 19077574]

[28] Stelzle F, Benner KU. Evaluation of different methods of indirect sinus floor elevation for elevation heights of $10 \mathrm{~mm}$ : An experimental ex vivo study. Clin Implant Dent Relat Res 2011; 13(2): 124-33. [http://dx.doi.org/10.1111/j.1708-8208.2009.00190.x] [PMID: 1968 1924]

[29] Sohn DS, Lee JS, An KM, Romanos GE. Erbium, chromium:yttriumscandium-gallium-garnet laser-assisted sinus graft procedure. Lasers Med Sci 2009; 24(4): 673-7.

[http://dx.doi.org/10.1007/s10103-009-0645-8] [PMID: 19219483]

[30] Mark Lin. Controlled technique for indirect sinus grafting with simultaneous implant placement.Oral Health J Dent Pract Manage 2013; 01: 1-8.

[31] Peñarrocha-Diago M, Galán-Gil S, Carrillo-García C, PeñarrochaDiago D, Peñarrocha-Diago M. Transcrestal sinus lift and implant placement using the sinus balloon technique. Med Oral Patol Oral Cir Bucal 2012; 17(1): e122-8.

[http://dx.doi.org/10.4317/medoral.17268] [PMID: 22157670]

[32] Peñarrocha-Diago $M$, Peñarrocha-Diago $M$, Sanchez-Recio $C$, Peñarrocha-Oltra D, Romero-Millán J. Osteotomy in direct sinus lift. A comparative study of the rotary technique and ultrasound. Med Oral Patol Oral Cir Bucal 2012; 17(3): e457-61.

[http://dx.doi.org/10.4317/medoral.17599] [PMID: 22143735]

[33] Chan HL, Oh TJ, Fu JH, Benavides E, Avila-Ortiz G, Wang HL. Sinus augmentation via transcrestal approach: A comparison between the balloon and osteotome technique in a cadaver study. Clin Oral Implants Res 2013; 24(9): 985-90.

[PMID: 22725990]

[34] Pozzi A, Moy PK. Minimally invasive transcrestal guided sinus lift (TGSL): A clinical prospective proof of concept cohort study up to 52 months. Clin Implant Dent Relat Res 2014; 16(4): 582-93. [http://dx.doi.org/10.1111/cid.12034] [PMID: 23356732]

[35] Bernardello F, Righi D, Cosci F, Bozzoli P, Soardi CM, Spinato S. Crestal sinus lift with sequential drills and simultaneous implant placement in sites with $<5 \mathrm{~mm}$ of native bone: A multicenter retrospective study. Implant Dent 2011; 20(6): 439-44. [http://dx.doi.org/10.1097/ID.0b013e3182342052] [PMID: 21989240]

[36] Wen SC, Lin YH, Yang YC, Wang HL. The influence of sinus membrane thickness upon membrane perforation during transcrestal sinus lift procedure. Clin Oral Implants Res 2015; 26(10): 1158-64. [http://dx.doi.org/10.1111/clr.12429] [PMID: 24891094]

[37] Jodia K, Sadhwani BS, Parmar BS, Anchlia S, Sadhwani SB. Sinus elevation with an alloplastic material and simultaneous implant placement: A 1-stage procedure in severely atrophic maxillae. J Maxillofac Oral Surg 2014; 13(3): 271-80. [http://dx.doi.org/10.1007/s12663-013-0536-1] [PMID: 25018600]

[38] Hatano N, Shimizu Y, Ooya K. A clinical long-term radiographic evaluation of graft height changes after maxillary sinus floor augmentation with a 2:1 autogenous bone/xenograft mixture and simultaneous placement of dental implants. Clin Oral Implants Res 2004; 15(3): 339-45.

[http://dx.doi.org/10.1111/j.1600-0501.2004.00996.x] [PMID: 1514 2097]

[39] Pjetursson BE, Ignjatovic D, Matuliene G, Brägger U, Schmidlin K, Lang NP. Transalveolar maxillary sinus floor elevation using osteotomes with or without grafting material. Part II: Radiographic tissue remodeling. Clin Oral Implants Res 2009; 20(7): 677-83. [http://dx.doi.org/10.1111/j.1600-0501.2009.01721.x] [PMID: 19515 059]

[40] Balleri P, Veltri M, Nuti N, Ferrari M. Implant placement in combination with sinus membrane elevation without biomaterials: A 1-year study on 15 patients. Clin Implant Dent Relat Res 2012; 14(5): 682-9.

[http://dx.doi.org/10.1111/j.1708-8208.2010.00318.x] [PMID: 2117 6096]

[41] Wallace SS, Froum SJ, Cho SC, et al. Sinus augmentation utilizing anorganic bovine bone (Bio-Oss) with absorbable and nonabsorbable membranes placed over the lateral window: Histomorphometric and clinical analyses. Int J Periodontics Restorative Dent 2005; 25(6): 551-9.

[PMID: 16353530]

[42] Choi KS, Kan JY, Boyne PJ, Goodacre CJ, Lozada JL, Rungcharassaeng $\mathrm{K}$. The effects of resorbable membrane on human maxillary sinus graft: A pilot study. Int J Oral Maxillofac Implants 2009; 24(1): 73-80. [PMID: 19344028]

[43] Jensen T, Schou S, Stavropoulos A, Terheyden H, Holmstrup P. Maxillary sinus floor augmentation with Bio-Oss or Bio-Oss mixed with autogenous bone as graft: A systematic review. Clin Oral Implants Res 2012; 23(3): 263-73.

[http://dx.doi.org/10.1111/j.1600-0501.2011.02168.x] [PMID: 21443 592]

[44] Jensen T, Schou S, Gundersen HJ, Forman JL, Terheyden H, Holmstrup P. Bone-to-implant contact after maxillary sinus floor augmentation with Bio-Oss and autogenous bone in different ratios in mini pigs. Clin Oral Implants Res 2013; 24(6): 635-44. [http://dx.doi.org/10.1111/j.1600-0501.2012.02438.x] [PMID: 22530 746]

[45] Proussaefs P, Lozada J, Kim J, Rohrer MD. Repair of the perforated sinus membrane with a resorbable collagen membrane: A human study. Int J Oral Maxillofac Implants 2004; 19(3): 413-20. [PMID: 15214227]

[46] Hernández-Alfaro F, Torradeflot MM, Marti C. Prevalence and management of Schneiderian membrane perforations during sinus-lift procedures. Clin Oral Implants Res 2008; 19(1): 91-8. [PMID: 17961185]

[47] Ardekian L, Oved-Peleg E, Mactei EE, Peled M. The clinical significance of sinus membrane perforation during augmentation of the maxillary sinus. J Oral Maxillofac Surg 2006; 64(2): 277-82. [http://dx.doi.org/10.1016/j.joms.2005.10.031] [PMID: 16413901]

[48] Schwartz-Arad D, Herzberg R, Dolev E. The prevalence of surgical 
complications of the sinus graft procedure and their impact on implant survival. J Periodontol 2004; 75(4): 511-6.

[http://dx.doi.org/10.1902/jop.2004.75.4.511] [PMID: 15152813]

[49] Zijderveld SA, van den Bergh JP, Schulten EA, ten Bruggenkate CM. Anatomical and surgical findings and complications in 100 consecutive maxillary sinus floor elevation procedures. J Oral Maxillofac Surg 2008; 66(7): 1426-38.

[http://dx.doi.org/10.1016/j.joms.2008.01.027] [PMID: 18571027]

[50] Al-Almaie S. Staged osteotome sinus floor elevation for progressive site development and immediate implant placement in severely resorbed alveolar bone: A case report. Case Rep Dent 2013; 2013310931

[http://dx.doi.org/10.1155/2013/310931] [PMID: 24251045]
[51] Hallman M, Sennerby L, Zetterqvist L, Lundgren S. A 3-year prospective follow-up study of implant-supported fixed prostheses in patients subjected to maxillary sinus floor augmentation with a 80:20 mixture of deproteinized bovine bone and autogenous bone Clinical, radiographic and resonance frequency analysis. Int J Oral Maxillofac Surg 2005; 34(3): 273-80. [http://dx.doi.org/10.1016/j.ijom.2004.09.009] [PMID: 15741036]

[52] Johansson LA, Isaksson S, Lindh C, Becktor JP, Sennerby L. Maxillary sinus floor augmentation and simultaneous implant placement using locally harvested autogenous bone chips and bone debris: A prospective clinical study. J Oral Maxillofac Surg 2010; 68(4): 837-44.

[http://dx.doi.org/10.1016/j.joms.2009.07.093] [PMID: 20307768]

\section{C) 2019 Al-Almaie and Kavarodi}

This is an open access article distributed under the terms of the Creative Commons Attribution 4.0 International Public License (CC-BY 4.0), a copy of which is available at: (https://creativecommons.org/licenses/by/4.0/legalcode). This license permits unrestricted use, distribution, and reproduction in any medium, provided the original author and source are credited. 